
\title{
R Research foure \\ The Effect of Pelvic Movements of a Gait Training System for Stroke Patients
}

\section{Choonghyun Son}

Korea Institute of Science and Technology

\section{Anna Lee}

Asan Medical Center

Junkyung Lee

Hallym University Dongtan Sacred Heart Hospital

DaeEun Kim

Yonsei University

\section{Seung-Jong Kim}

Korea University - Seoul Campus: Korea University

\section{Min Ho Chun}

Asan Medical Center

Junho Choi ( $\square$ junhochoi@kist.re.kr)

The Center for Bionics, Korea Institute of Science and Technology https://orcid.org/0000-0003-42694787

\section{Research}

Keywords: Rehabilitation, Stroke, Exoskeleton, Gait training, Pelvic movement

Posted Date: March 1st, 2021

DOI: https://doi.org/10.21203/rs.3.rs-254165/v1

License: (c) (1) This work is licensed under a Creative Commons Attribution 4.0 International License.

Read Full License 


\title{
RESEARCH
}

\section{The effect of pelvic movements of a gait training system for stroke patients}

\author{
Choonghyun Son ${ }^{1,3}$, Anna Lee ${ }^{2}$, Junkyung Lee ${ }^{4}$, DaeEun Kim ${ }^{3}$, Seung-Jong Kim ${ }^{5}$, Min Ho Chun ${ }^{2 \dagger}$ and \\ Junho $\mathrm{Choi}^{*} \dagger$
}

\begin{abstract}
Background: Aging societies lead to higher demand for gait rehabilitation as age-related neurological disorders such as stroke increase. Since conventional methods for gait rehabilitation are physically and economically burdensome, robotic gait training systems have been studied and commercialized, many of which provided movements confined in the sagittal plane. For better outcomes of gait rehabilitation with more natural gait patterns, however, it is desirable to provide pelvic movements in the transverse plane. In this study, a robotic gait training system capable of pelvic motions in the transverse plane was used to evaluated the effect of the pelvic motions on stroke patients.
\end{abstract}

Method: Healbot $\mathrm{T}$, which is a robotic gait training system and capable of providing pelvic movements in the transverse plane as well as flexion/extension of the hip and knee joints and adduction/abduction of the hip joints, is introduced and used to evaluate the effect of the pelvic movement on gait training of stroke patients.

Experiment: 23 stroke patients with hemiparesis participated in this study and were assigned into two groups. Pelvis-on group was provided with pelvic motions whereas no pelvic movement was allowed for pelvis-off group during 10 sessions of gait trainings in Healbot T. EMG signals and interaction forces as well as the joint angles of the robot were measured. Gait parameters such as stride length, gait period, cadence, and walking speed were measured after gait training.

Result: $37.5 \%$ lower interaction forces of pelvis were observed in the pelvis-on group than the pelvis-off group. Furthermore, the interaction forces at the thighs and calves of both groups showed significant decrease. The EMG signals of gluteus medius of the pelvis-on group increased by $77.2 \%$. Furthermore, statistically significant increases in various muscles were measured in the pelvis-on group during the stance phase.

Conclusion: Gait training using a robotic gait training system with pelvic movements was conducted to study the effects of lateral and rotational pelvic movements in gait training of stroke patients. The pelvic movements made gait training less interfered by the exoskeleton while stimulating the voluntary muscle activation during the stance phase.

Clinical trial registration: KCT0003762, 2018-1254, Registered 28 October 2018, https://cris.nih.go.kr/cris/search/search_result_st01_kren.jsp?seq=14310\&ltype =\&rtype=

Keywords: Rehabilitation; Stroke; Exoskeleton; Gait training; Pelvic movement

\footnotetext{
${ }^{*}$ Correspondence:

junhochoi@kist.re.kr

${ }^{1}$ The Center for Bionics, Korea Institute of Science and Technology, 5, Hwarang-ro 14-gil, Seungbuk-gu, 02792 Seoul, Republic of Korea

Full list of author information is available at the end of the article ${ }^{\dagger}$ Equal contributor
}

\section{Introduction}

As society aged, the number of stroke patients increases [1]. By 2030, 70 million stroke patients and 12 million deaths by stroke in the world were anticipated [2,3]. As a consequence, increased number of stroke patients have suffered from loss of gait function, which is essential for the activities of daily living (ADL's) [4].

Conventional therapies to recover the gait function impose physical load to the therapists as well as economical burden to the patients. In addition, the outcomes of gait training are often limited by inaccurate gait patterns and incorrect assessment of the patients $[5,6]$. In order to reduce the burdens, robotic gait training systems 
have been studied and used [7, 8]. These robotic gait training systems effectively increased the time and intensity of training, which is crucial for better rehabilitation outcomes. Despite the increased time and intensity of training, however, reduced muscle activation was observed if the subjects remained completely passive in those gait training systems [9]. In order to overcome this drawback, several researches have improved the control methods to increase the level of participation of the patients by giving the patients a certain amount of freedom to change trajectories of the gait training systems [10].

Further improvements have been made to include pelvic movements in the transverse plane, which were excluded in the early designs due to higher complexity and costs to build the gait training systems. However, since lateral and rotational pelvic movements, which are two of the six determinants of walking [11], are responsible for balancing and weight-shifting during walking and increasing stride length $[12,13]$, it is natural to include those movements for better gait training. Therefore, the lateral and rotational pelvic movements have been added in recently developed robotic gait training systems. Banala et al. added passive joints to a gait training system to allow lateral and rotational pelvic movements [14, 15]. The passive joints including the ones allowing lateral and rotational movements in the transverse plane was held with springs to allow pelvic movements during gait training. In other cases, series elastic actuators were used to actuate an exoskeleton capable of providing transnational and lateral movements in the transverse plane as well as joint rotation in the sagittal plane $[16,17,18]$. The experiments involving healthy subjects in these systems showed similar muscle activation compared to treadmill walking. However, the effects of the pelvic movements were not studied in depth. FreeD, which was an optional module to allow pelvic movements, was added to the Lokomat to accommodate more physiological gait patterns $[19,20]$. When compared to the case without the module, less compensatory movements of the trunk were observed when the pelvic motion was added. Hence, more natural gait patterns was obtained during training with less compensatory movement of the trunk.

In this study, Healbot $\mathrm{T}$, which is a gait training system capable of providing pelvic movements, was introduced. The concept and feasibility of Healbot $\mathrm{T}$ were introduced in the previous studies and the effect of the pelvic movements on healthy people using a gait training system called COWALK-I was presented in [21, 22]. Healbot $\mathrm{T}$, which is developed and improved from COWALK-I, was used to study the effect of pelvic movements during gait training of stroke patients with hemiparesis. The patients were divided into two groups and asked to walk in Healbot T. During gait trainings, one group was provided with pelvic movements whereas the other group walked without pelvic movements. The group with pelvic motions showed increased muscle activation during the stance phase and less interaction forces at the pelvis. Both groups showed decreased interaction forces at the thighs and calves. This result implies that the group with pelvic movements have more voluntary muscle activation.

\section{Method}

Protocol

23 subjects with hemiparesis from stroke visited Asan Medical Center (AMC) 10 times within one month. During a visit, subjects were asked to walk in Healbot T, 
which was a robotic gait training system designed to provide pelvic movements in the transverse plane as well as joint movements in the sagittal plane. It consisted of an exoskeleton, a gravity compensator, a body weight support (BWS) system, and foot plates. Figure 1 shows a subject in the Healbot $\mathrm{T}$ during a gait training. Detailed description of the Healbot $\mathrm{T}$ is explained in a later section.

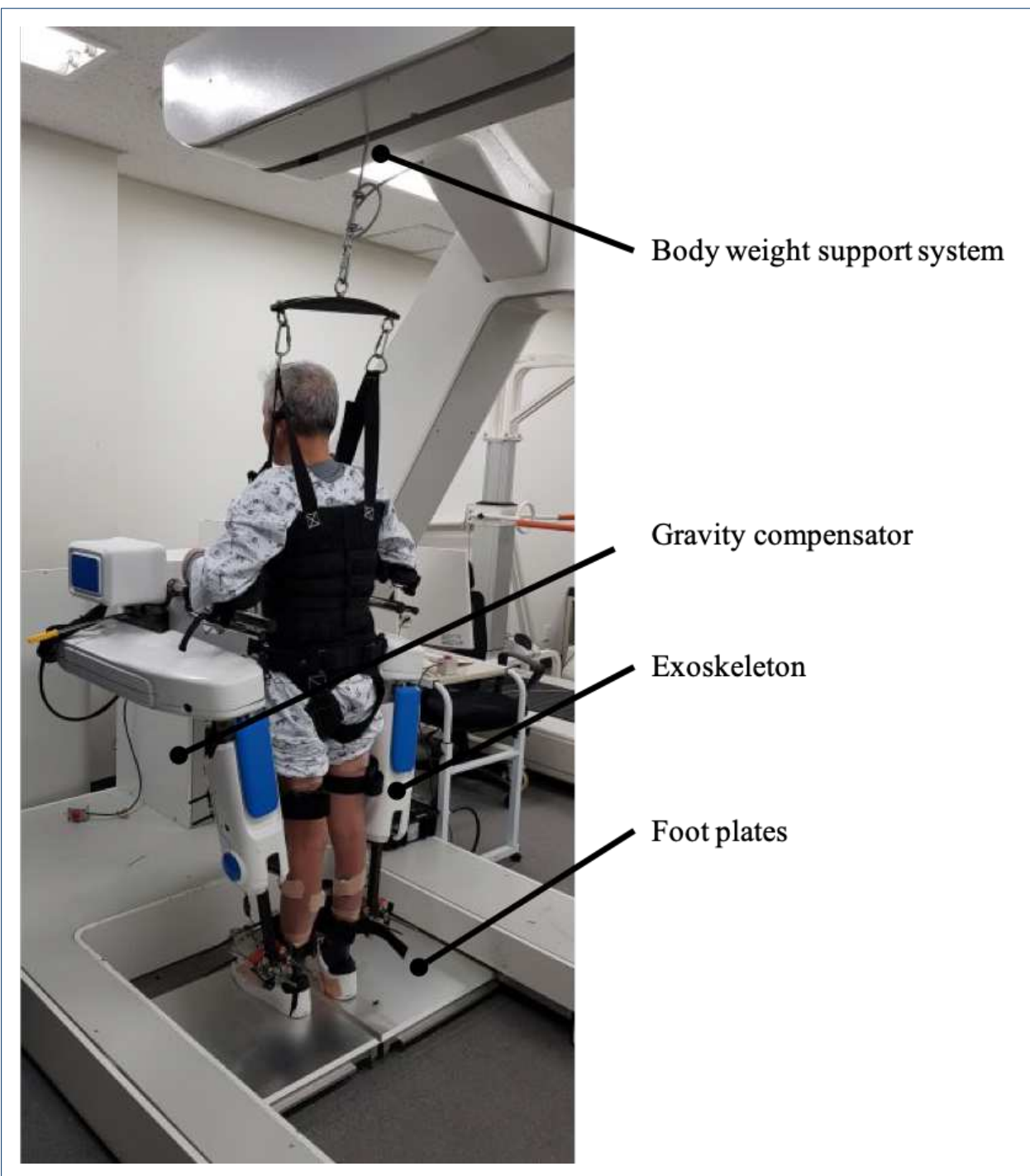

Figure 1 Subject in the Healbot $\mathrm{T}$ during training

The subjects were randomly assigned into two groups, which were pelvis-off and pelvis-on groups. For the pelvis-off group, pelvic movements in the transverse plane were not allowed during gait trainings, whereas transnational and rotational pelvic movements in the transverse plane were provided for the pelvis-on group. Prior to the gait trainings, the length of each body segment of the subjects was measured and used to adjust the length of the corresponding link of the exoskeleton.

Each training session lasted 30 minutes, during which the subjects walked in the Healbot T. The exoskeleton was attached to the subjects using braces and straps at the pelvis, thighs, and calves. If necessary, the BWS system was used to support the 
weight of the subjects. Predefined trajectories, which were collected from a healthy person on a treadmill prior to this experiment, were used to control the exoskeleton. Note that the trajectories of the pelvic joints for the pelvis-off group are set to be constants. At the beginning of each session, preferred walking speeds were selected by the subjects. As the walking speed gradually increased from the initial walking speed of $0.5 \mathrm{~km} / \mathrm{h}$, the subjects selected a comfortable walking speed.

Interaction forces between the subjects and the exoskeleton were measured using force sensors (UMM31-K100, Dacell) installed at the braces during the trainings. Muscle activation of the gluteus medius (Gmed), rectus femoris (RF), tibialis anterior (TA), biceps femoris (BF), gastrocnemius medial (GCM-M), gastrocnemius lateral (GCM-L) muscles of both sides were measured using EMG sensors (Trigino, Delsys) during the trainings on the 1st, 5 th, and 10th days. Two custom-made foot pressure sensors using force sensitive resisters (FSR-402, Interlink) were attached beneath each sole to determine the gait phase. See Figure 2 for the locations of the sensors. Gait parameters such as stride length, gait period, cadence, and walking speed were measured using OptoGait (Microgate) on the 1st, 5th, 10th days after the trainings followed by a 20 -minute cool-down period.
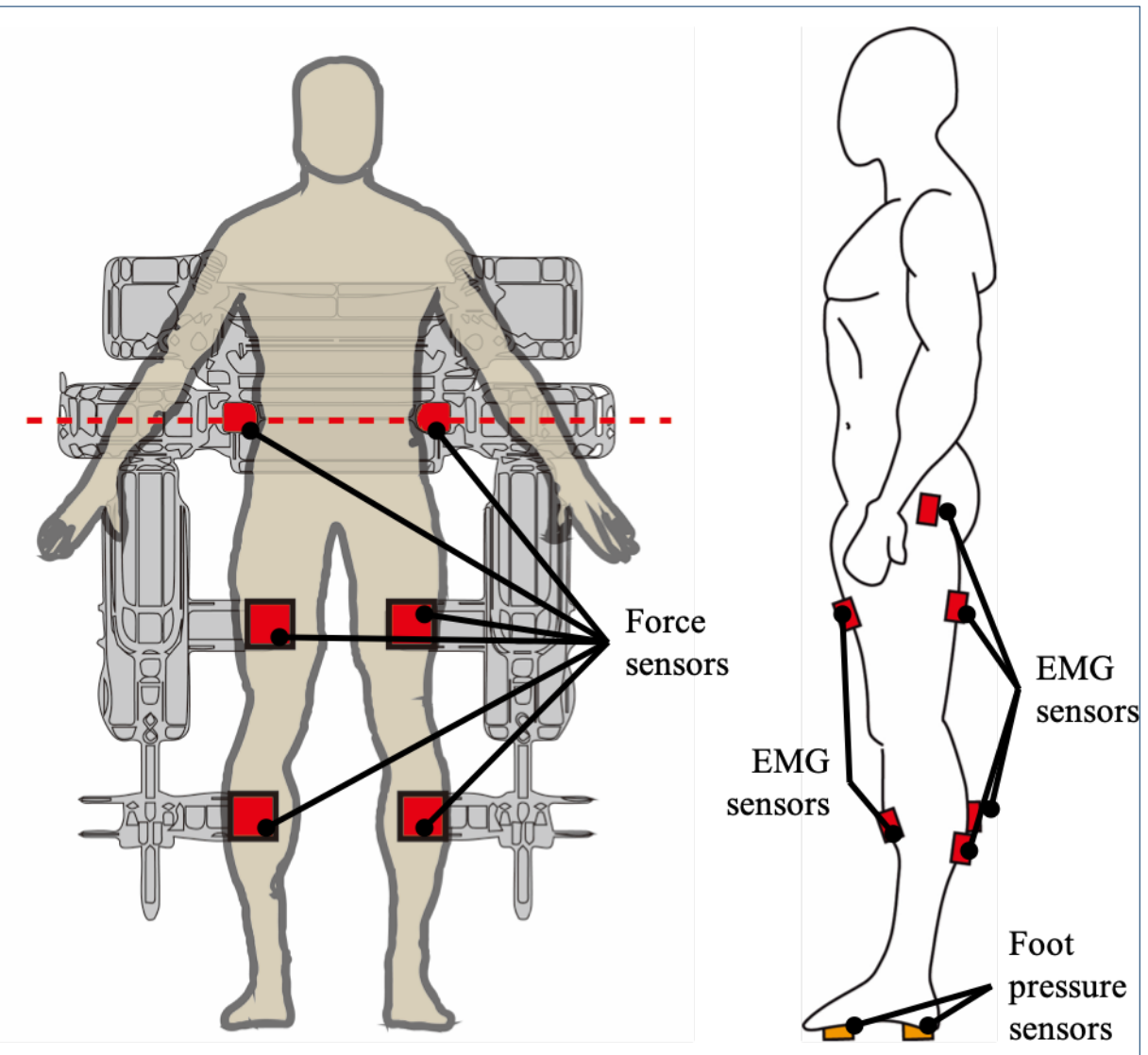

Figure 2 Locations of sensors. 6 force sensors are at the pelvis, thigh, and calf of each side. 12 EMG sensors are at the gluteus medius, rectus femoris, biceps femoris, tibialis anterior, gastrocnemius medial, gastrocnemius lateral. 2 pressure sensors are beneath each foot. 
Patient information

All 23 participants were informed of the study objectives and procedures prior to signing a informed consent form. The study was approved by the Asan Medical Center Institutional Review Board (IRB, No. 2018-1254). Detailed information on the subjects is listed in Table 1. No statistical differences were found in weights, age, duration of onset, and Functional Ambulation Category (FAC) between both groups. However, the height of the pelvis-on group is greater than the pelvis-off group $(\mathrm{p}<0.05)$.

Table 1 Patient information

\begin{tabular}{ccc}
\hline & Pelvis-off & Pelvis-on \\
\hline \# of subject & $11(\mathrm{M}: 4, \mathrm{~F}: 7)$ & $12(\mathrm{M}: 10, \mathrm{~F}: 2)$ \\
Age (years) & $66.7(14.5)$ & $66.4(7.9)$ \\
Onset period (years) & $14.5(6.1)$ & $15.8(8.1)$ \\
FAC (score) & $4.00(0.63)$ & $4.58(1.2)$ \\
Height (cm)** & $158.0(6.9)$ & $165.4(6.8)$ \\
Weight (kg) & $65.1(8.9)$ & $68.0(8.2)$ \\
\hline
\end{tabular}

\section{Result}

Statistical analysis

Due to large amount of steps, the interaction forces and the EMG signals, which were averaged over steps, were considered to have normal distributions. The interaction force, EMG signals and gait parameters were compared using a two-tailed T-test. Three different significance levels were denoted by $*(\mathrm{p}<0.1),{ }^{* *}(\mathrm{p}<0.05)$, *** $(\mathrm{p}<0.01)$ marks.

\section{Self-selected speed}

The measured self-selected walking speeds in every session are shown in Figure 3. The self-selected walking speed gradually increased as the training progressed. However, there was no significant difference between both groups.

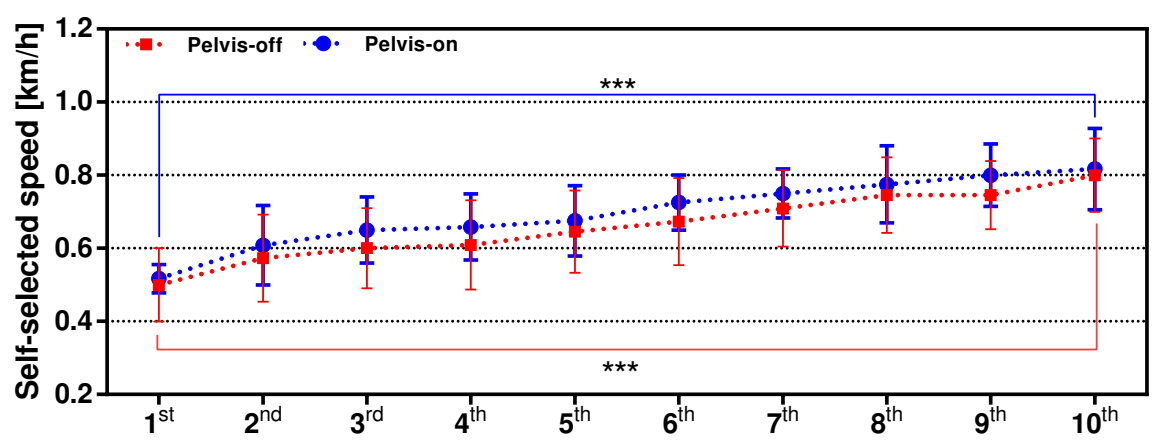

Figure 3 The self-selected speed of both groups. The red and blue lines represent the averaged self-selected speeds of the pelvis-off group and the pelvis-on group, respectively.

Interaction force

The interaction forces were measured using the force sensors installed on the braces at pelvis, thighs, and calves as shown in Figure 2. Note that two force sensors 
were required to measure the interaction force at the pelvis since unilateral force (i.e. pushing against the sensor) was applied to the sensors. Therefore, the net interaction forces were calculated as $f_{\text {pelvis }}=f_{\mathrm{A}}+f_{\mathrm{U}}$, where $f_{\mathrm{A}}$ represented the interaction force at the affected side $(\mathrm{A})$ of the pelvis and $f_{\mathrm{U}}$ denoted the interaction force at the unaffected side $(\mathrm{U})$.

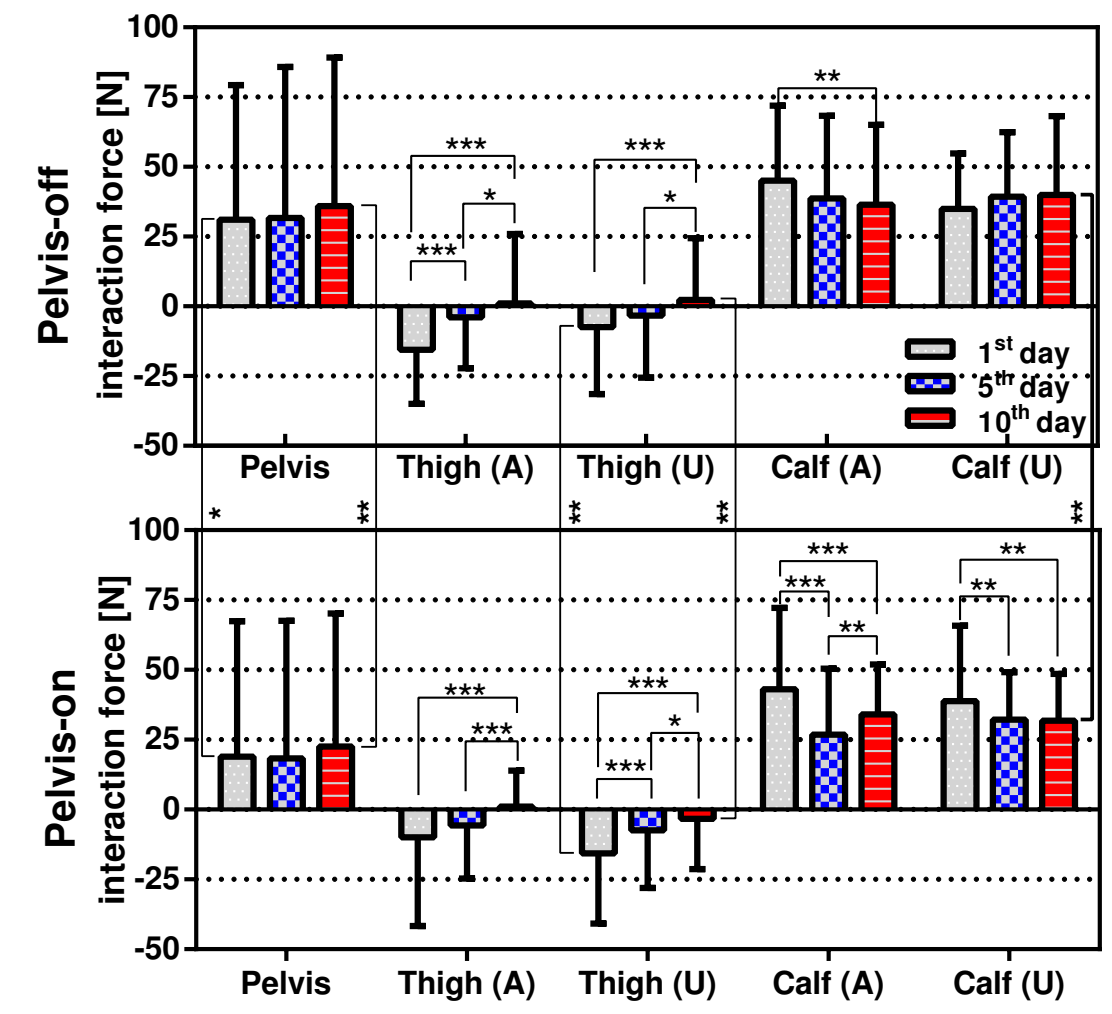

Figure 4 Measured interaction forces. The bar graphs represent mean of interaction force in both groups. The error bars represent standard deviation of interaction forces in both groups.

The measured interaction forces are shown in Figure 4. No significant changes between the first and last days of the training in both groups was found. Positive interaction forces at the pelvis indicated that the patients leaned toward the affected side, which were observed in both groups. However, interaction forces measured at the pelvis of the pelvis-on group was $37.5 \%$ lower than the pelvis-off group on the 10th day. Interaction forces at the other locations except the calf of the unaffected side of the pelvis-off group significantly decreased on the 10th day.

\section{EMG signal}

The measured EMG signals were filtered with a band pass filter with pass band frequencies of $20 \sim 500 \mathrm{~Hz}$, rectified, and normalized by their maximum values.

As shown in Figure 5, there is no significant difference in EMG signals between both of the groups on the 1st day. However, the mean EMG signals of the pelvis- 

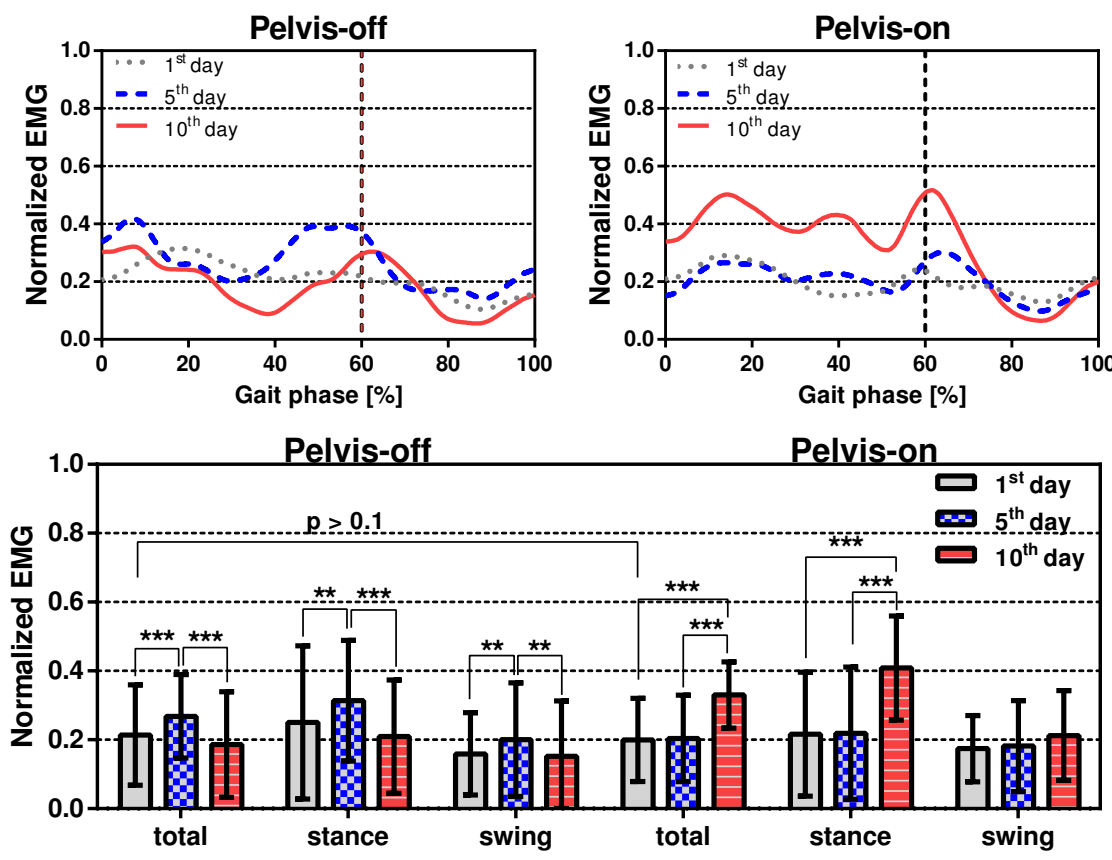

Figure 5 Normalized EMG records of Gmed on the affected side of both groups are at the top. Vertical dashed lines indicate the toe-off. The EMG records during different gait phases are at the bottom. The error bars represent standard deviation of EMG signal

on group increased by $77.2 \%$ on the last day of training. In particular, the EMG signals during stance phase rose $95.2 \%$ in the pelvis-on group.

The mean EMG signals of 4 other muscles in the affected side of the pelvis-on group, which were RF, TA, BF, and GCM-L, increased by $51.0 \%, 15.5 \%, 31.4$ $\%$, and $135.7 \%$, respectively, during the experiment as shown in Figure 6. In the pelvis-off group, the EMG signal of RF and GCM-L increased by $24.8 \%, 32.6 \%$ on the 10th day. Furthermore, the EMG signals at RF, TA, Gmed, BF, and GCM-L muscles showed significant differences between both groups on the last day of the experiment.

\section{Gait parameters}

Figure 7 shows the gait parameters that were measured using OptoGait, which were stride length, walking period, cadence and walking speed. Each parameter was normalized by the data measured on the 1st day. No significant differences between the pelvis-off and pelvis-on group and between training sessions was found in stride length, gait period, walking speed. However, significant difference was found in cadence between both groups on the 10th day.

\section{Healbot T}

Healbot $\mathrm{T}$ was a gait training system designed for patients with stroke. It consisted of an exoskeleton robot, a BWS system, a gravity compensator, and footplates, see Figure 8. 

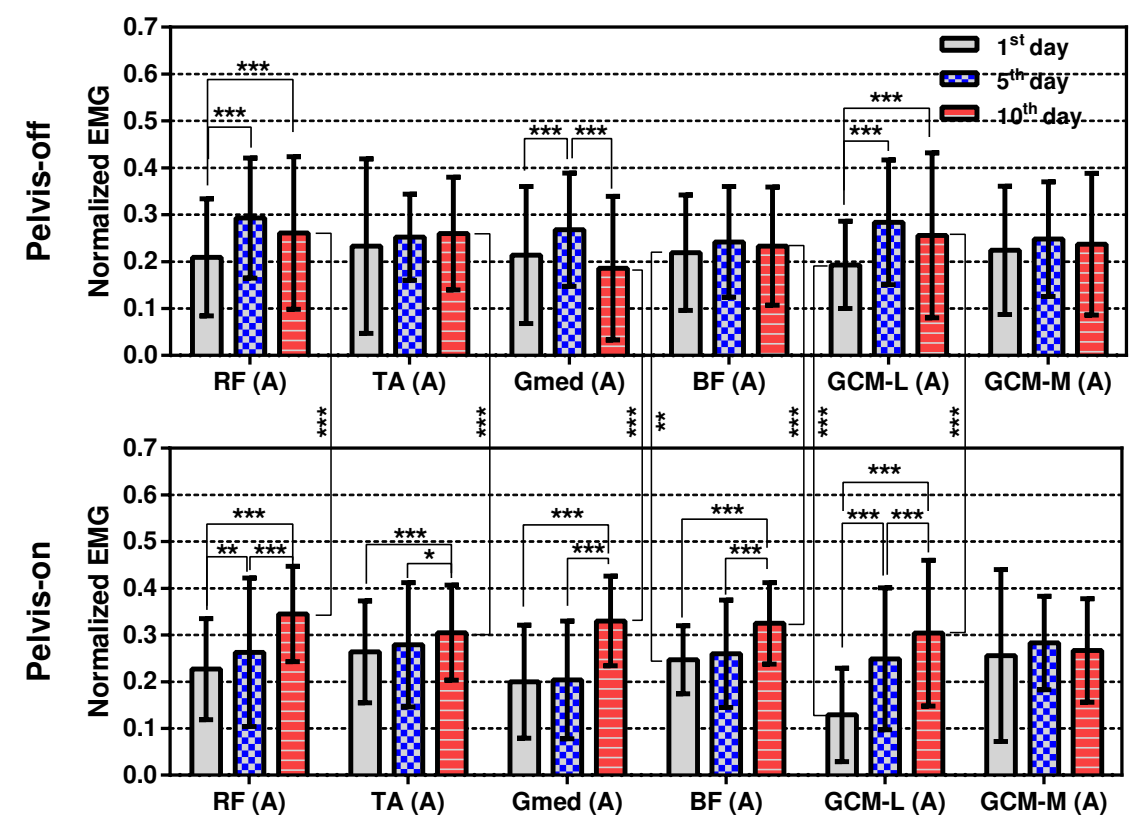

Figure 6 Averaged EMG signals of the 6 muscles on the affected sides of both groups. Top is the EMG signals of the pelvis-off group and bottom is the EMG signal of the pelvis-on group.
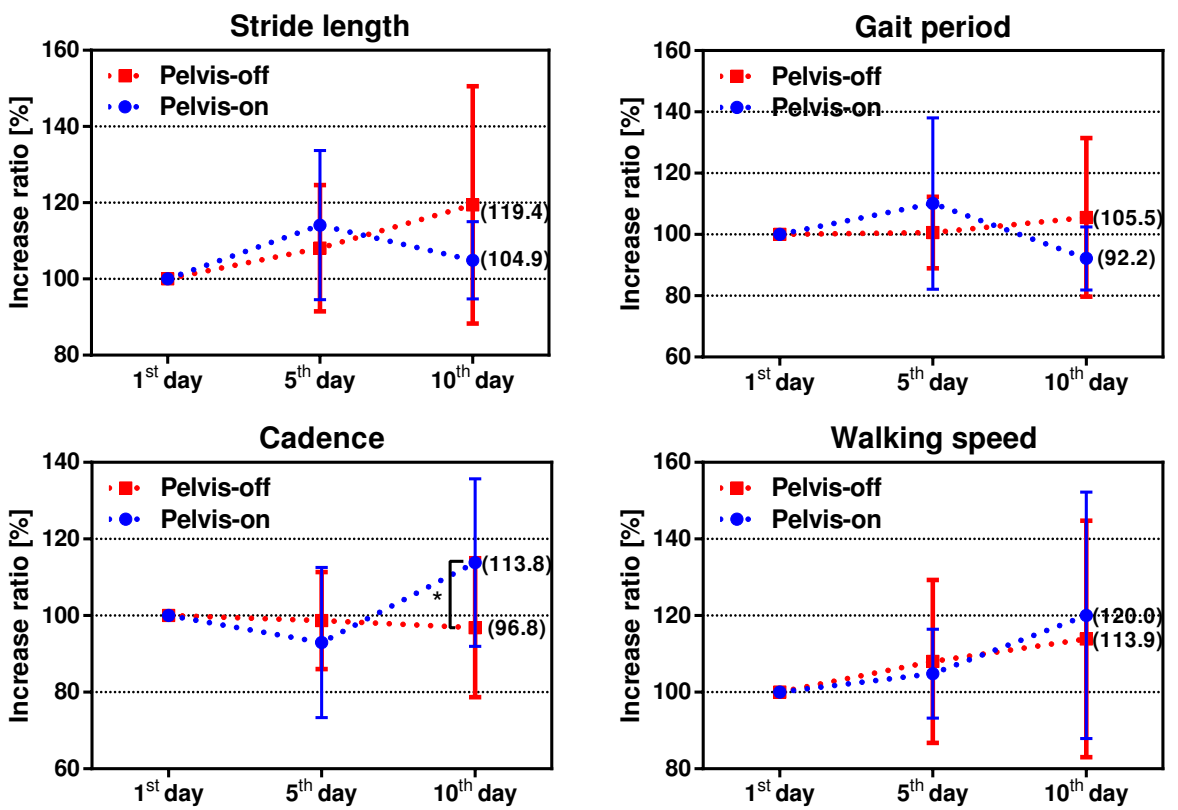

Figure 7 Measured gait parameters. Red and blue lines represent the pelvis-off group and the pelvis-on group. Error bars showed standard deviation. 


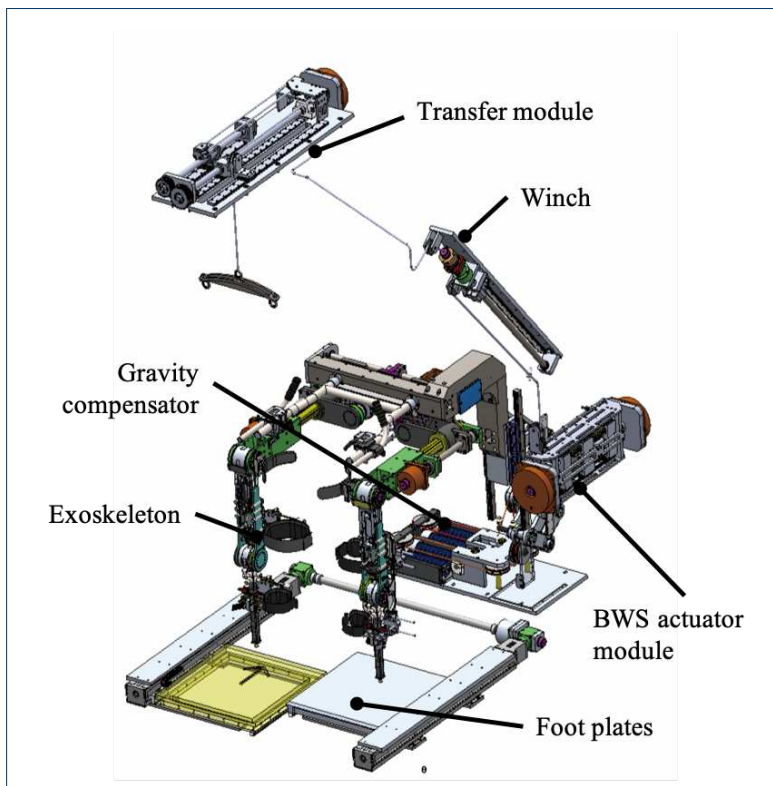

(a)

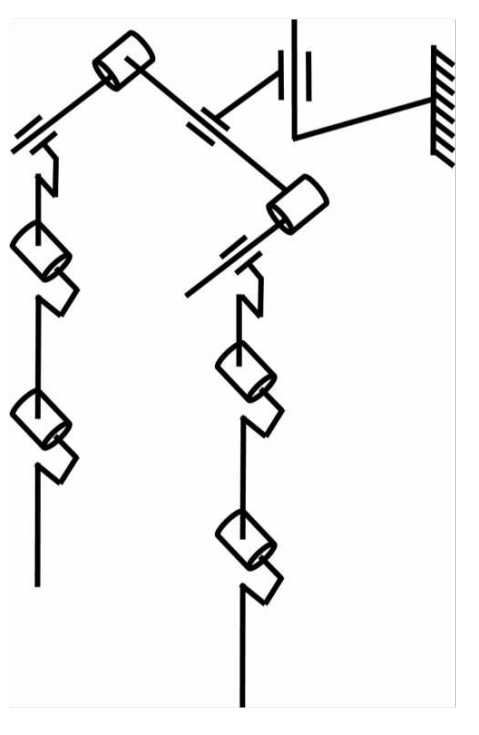

(b)

Figure 8 (a) Designed Healbot T. Healbot T consists of a lower limb exoskeleton robot with 9 active DoF and 1 passive DoF, a BWS system, a gravity compensator, and foot plates. (b) Schematics of Healbot $T$, which has 9 active joints and 1 passive joint for vertical displacement

The exoskeleton robot was designed to provide pelvic movements. It had 9 active joints, which were three prismatic joints for transnational and rotational movements in the transverse plane and six rotational joints for adduction/abduction at the hip joint and extension/flexion of the hip and knee joints, and 1 passive joint for vertical displacement. For the rotational joints, six identical BLDC motors (K064050-EY2, Parker) with harmonic gear reducers (SHG-20-100-2SO, HDS) were used. Three prismatic joints were actuated with three identical BLDC motors (K089200-7Y2, Parker) with lead-screws to generate lateral, forward, and rotational pelvic movements in the transverse plane. Positions of the actuated joints were measured using absolute encoders (EQI-1131, Heidenhain) attached to the shaft of the motors.

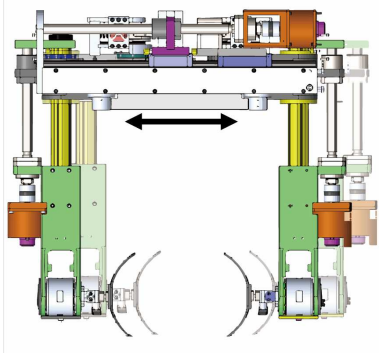

(a)

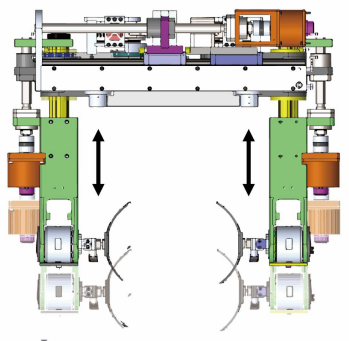

(b)

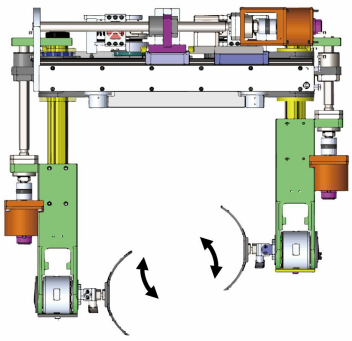

(c)

Figure 9 Pelvic movement generated with three prismatic joints (a) Lateral movement (b) Forward movement (c) Rotational movement 
Pelvic movements in the transverse plane were generated using three prismatic joints. Lateral movement was generated by the prismatic joint in the center of the exoskeleton. Forward and rotational movements were produced by the prismatic joints at both sides. See Figure 9.

The braces for securing the patients are located at the pelvis, thighs and calves of the exoskeleton. Force sensors are installed between the exoskeleton and the braces to measure the interaction forces. The footplates were controlled to match the patient's foot position. Each joint angles are kept within a preset range of motion by mechanical and programmable limits for safety.

The BWS system is designed for supporting the subjects having difficulty in controlling their trunk positions. The BWS system consists of an actuator module, a winch, and a transfer module as shown in Figure 8.

Since the weight of the exoskeleton imposes physical burden to the subjects wearing the exoskeleton robot and adverse effect on the gait [23], a gravity compensator was designed to support the weight of the exoskeleton. The weight of the exoskeleton was supported by linear springs instead of counter mass to reduce the inertial effect on the exoskeleton. Using a 4-bar linkages and cable, the supporting force generated by the springs remained constant regardless of the position of the exoskeleton.

\section{Discussion}

The effect of pelvic movements during gait training was studied in this study. In order to provide gait patterns including pelvic movements, a robotic gait training system, which was called Healbot $\mathrm{T}$ and capable of pelvic movements, was developed. 23 stroke patients participated in the experiment and were divided into two groups. The pelvis-on group was provided with pelvic movements whereas no pelvic movement was given to the pelvis-off group during gait trainings in Healbot T.

The interaction forces at the pelvis of the pelvis-on group was lower than the pelvis-off group. Since one of the reasons for the interaction forces was the limited degrees of freedom of the exoskeleton, constrained pelvic movements imposed by the exoskeleton resulted in higher interaction forces at the pelvis. In contrast, the rotational and lateral pelvic movements contributed to reduce the interaction forces at the pelvis of the pelvis-on group. Positive values of the interaction forces indicated that both group leaned toward affected sides, which might be the result of poor trunk control during the trainings. Lower level of the interaction forces at the pelvis of the pelvis-on group showed less leaning of the torso toward the affected side.

Significant decrease of interaction forces at thighs and calves of both groups implied less interference from mismatched trajectories of the robot and the subject as well as less potential of injury and enhancing comfort [24]. Jezernik et al. showed that, when the trajectories provided by a gait training system were allowed to be adapted to the subjects, the interaction forces decreased as the trajectories were changed whereas the interaction forces remain high without adaptation [25]. In our study, significantly decreased interaction forces implies that both groups had adapted to the gait patterns provided by the gait training systems since the trajectories of hip and knee joints were not changed during a gait training.

Muscle activation of gluteus medius (Gmed) in Figure 5 showed no significant difference between 1st and 10th days in the pelvis-off group whereas the pelvis-on 
group showed increased muscle activation, which was not consistent with what was reported in the previous study [20]. The difference might be caused by the trajectories with larger lateral displacement provided by the exoskeleton. In the pelvis-off group, Gmed muscles were activated around toe-off, which was not observed in the experiments with healthy subjects $[17,18,20,26]$ but consistent with the experiments with stroke patients by van Kamman [27]. The muscle activation around toe-off of the swing leg might occur due to the effort to create the circumduction, which was often observed in the gait patterns of the stroke patients or weight shifting during the double stance phase since Gmed contributes to weight shifting [28].

No significant difference in Gmed muscle activation in the pelvis-off group was observed whereas increased muscle activation in the pelvis-on group were found during the stance phase, which is consistent with what was reported [27, 26, 29]. This implies that the muscle activation occurred due to voluntary balancing and weight bearing during the stance phase. Therefore, it is important to have lateral pelvic movements in gait training to improve balancing and weight shifting since Gmed muscles take a critical role in balancing and weight shifting during walking [30].

In the previous research by Hidler and Wall [26], increased activation in quadriceps muscles such as rectus femoris and gluteus muscle groups and decreased activation in gastrocnemius and tibialis anterior muscles were reported. However, in this study, only RF and GCM-L muscles of the pelvic-off group showed increased activation. Furthermore, all muscles except GCM-M showed increased activation in the pelvis-on group as shown in Figure 6. Since increased EMG signals indicates more voluntary muscle activation [31], increased muscle activation of the pelvis-on group implies voluntary muscle activation to support body weight with the affected leg during the trainings with pelvic motions.

It was observed that the cadence of the pelvis-on group increased while the pelvisoff group showed reduced cadence. Although statistical significance was not found, it is interesting to note that the walking speed of the both groups increased by increased stride length for the pelvis-off group and decreased gait period for the pelvis-on group. The result is consistent with Veneman [32] who reported increased step length of walking with fixed pelvis.

\section{Conclusion}

A robotic gait training system capable of providing pelvic movement, which was called Healbot T, was developed. The effects of translational and rotational pelvic movements on gait training of stroke patients were studied using Healbot T. The experiment was conducted with 23 stroke patients who were randomly assigned into two groups. The pelvis-off group was trained without pelvic movements while the pelvis-on group was provided with the pelvic movements during gait training using Healbot T. The pelvis-on group showed less interaction forces at the pelvis than the pelvis-off group. This indicates that Healbot $\mathrm{T}$ provided more natural and comfortable gait trajectories with lateral and rotating pelvic movement. Interaction forces at the thighs and calves decreased as training progressed in both groups, which implied gait adaption by both groups. Muscle activation of Gmed as well as $\mathrm{RF}, \mathrm{TA}, \mathrm{BF}$, and GCM-L muscles increased in the pelvis-on group. These results 
indicated that the pelvic motions provided by Healbot $\mathrm{T}$ induced voluntary muscle activation of the subjects for weight bearing.

\section{Declarations}

Ethics approval and consent to participate

All subjects have given written consent to the use of their data and information in this study. The protocol and process for this study was approved by Institutional Review Board (IRB) of Asan Medical Center (KCT0003762, 2018-1254). Also, this study was enrolled to Clinical Research information Service (CRIS) in S.Korea. This study was approved on 10 Oct, 2018. Patient trials were conducted after the clinical trial approval.

Consent for publication

All subjects and authors have consent for this publication.

Availability of data and materials

The datasets used and analysed in the manuscript are available from the corresponding author on reasonable request (Email: junhochoi@kist.re.kr).

\section{Competing interests}

The authors declare that they have no competing interests.

\section{Funding}

This work was supported by the Korea Institute of Science and Technology(KIST) Institutional Program (Project no. 2E31110), a grant (2018-314) from the Asan Institute for Life Sciences, Asan Medical Center, Seoul, Korea, and the National Research Foundation of Korea (NRF) grant funded by the Korea government (MSIT) (No.2020R1A2B5B01002395).

\section{Author's contributions}

C. S. conducted the experiment, performed statistical analysis, and drafted the manuscript. A. L. conducted the experiment and statistical analysis. D. K. designed the experiment. J. L and M. C. designed the experiment, analyzed the data, and examined the patients. S. K. designed the apparatus and experiment, J. C. conceived and managed the project and finalized the manuscript. All authors reviewed the manuscript.

Corresponding author

Correspondence to J. C.

\section{Acknowledgements}

Not applicable.

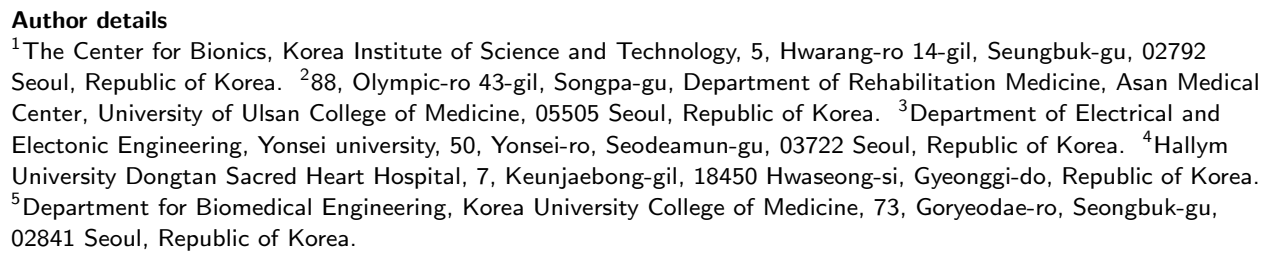


References

1. Benjamin, E.J., Muntner, P., Alonso, A., Bittencourt, M.S., Callaway, C.W., Carson, A.P., Chamberlain, A.M. Chang, A.R., Cheng, S., Das, S.R., Delling, F.N., Djousse, L., Elkind, M.S.V., Ferguson, J.F., Fornage, M., Jordan, L.C., Khan, S.S., Kissela, B.M., Knutson, K.L., Kwan, T.W., Lackland, D.T., Lewis, T.T., Lichtman, J.H., Longenecker, C.T., Loop, M.S., Lutsey, P.L., Martin, S.S., Matsushita, K., Moran, A.E., Mussolino, M.E., O'Flaherty, M., Pandey, A., Perak, A.M., Rosamond, W.D., Roth, G.A., Sampson, U.K.A., Satou, G.M., Schroeder, E.B., Shah, S.H., Spartano, N.L., Stokes, A., Tirschwell, D.L., Tsao, C.W., Turakhia, M.P., VanWagner, L.B., Wilkins, J.T., Wong, S.S., Virani, S.S., On behalf of the American Heart Association Council on Epidemiology and Prevention Statistics Committee and Stroke Statistics Subcommittee: Heart disease and stroke statistics-2019 update: A report from the american heart association. Circulation 139(10), 56-528 (2019). doi:10.1161/CIR.0000000000000659.

https://www.ahajournals.org/doi/pdf/10.1161/CIR.0000000000000659

2. Feigin, V.L., Forouzanfar, M.H., Krishnamurthi, R., Mensah, G.A., Connor, M., Bennett, D.A., Moran, A.E., Sacco, R.L., Anderson, L., Truelsen, T., et al.: Global and regional burden of stroke during 1990-2010: findings from the global burden of disease study 2010. The Lancet 383(9913), 245-255 (2014)

3. Ziegler-Graham, K., MacKenzie, E.J., Ephraim, P.L., Travison, T.G., Brookmeyer, R.: Estimating the prevalence of limb loss in the united states: 2005 to 2050. Archives of physical medicine and rehabilitation 89(3), 422-429 (2008)

4. Richards, C.L., Malouin, F., Wood-Dauphinee, S., Williams, J., Bouchard, J.-P., Brunet, D.: Task-specific physical therapy for optimization of gait recovery in acute stroke patients. Archives of physical medicine and rehabilitation 74(6), 612-620 (1993)

5. Chen, B., Ma, H., Qin, L.-Y., Gao, F., Chan, K.-M., Law, S.-W., Qin, L., Liao, W.-H.: Recent developments and challenges of lower extremity exoskeletons. Journal of Orthopaedic Translation 5, 26-37 (2016)

6. Viteckova, S., Kutilek, P., Jirina, M.: Wearable lower limb robotics: A review. Biocybernetics and biomedical engineering 33(2), 96-105 (2013)

7. Colombo, G., Joerg, M., Schreier, R., Dietz, V.: Treadmill training of paraplegic patients using a robotic orthosis. Journal of Rehabilitation Research \& Development 37, 693-700 (2000)

8. Fisher, S., Lucas, L., Adam Thrasher, T.: Robot-assisted gait training for patients with hemiparesis due to stroke. Topics in stroke rehabilitation 18(3), 269-276 (2011)

9. Israel, J.F., Campbell, D.D., Kahn, J.H., Hornby, T.G.: Metabolic costs and muscle activity patterns during robotic- and therapist-assisted treadmill walking in individuals with incomplete spinal cord injury. Physical Therapy 86(11), 1466-1478 (2006)

10. Duschau-Wicke, A., von Zitzewitz, J., Caprez, A., Lünenburger, L., Riener, R.: Path control: A method for patient-cooperative robot-aided gait rehabilitation. IEEE Transactions on Neural Systems and Rehabilitation Engineering 18(1), 38-48 (2010)

11. Saunders, J.B.d.M., Inman, V.T., Eberhart, H.D.: The major determinants in normal and pathological gait. The Journal of Bone \& Joint Surgery 35(3), 543-558 (1953)

12. Mun, K.-R., Guo, Z., Yu, H.: Restriction of pelvic lateral and rotational motions alters lower limb kinematics and muscle activation pattern during over-ground walking. Medical \& biological engineering \& computing 54(11), 1621-1629 (2016)

13. Wu, M., Kim, J., Arora, P., Gaebler-Spira, D.J., Zhang, Y.: The effects of the integration of dynamic weight shifting training into treadmill training on walking function of children with cerebral palsy: A randomized controlled study. American Journal of Physical Medicine \& Rehabilitation 96(11), 765-772 (2017)

14. Banala, S.K., Agrawal, S.K., Scholz, J.P.: Active leg exoskeleton (ALEX) for gait rehabilitation of motor-impaired patients. In: IEEE International Conference on Rehabilitation Robotics, pp. 401-407 (2007). IEEE

15. Banala, S.K., Kim, S.H., Agrawal, S.K., Scholz, J.P.: Robot assisted gait training with active leg exoskeleton (ALEX). IEEE Transactions on Neural Systems and Rehabilitation Engineering 17(1), 2-8 (2009)

16. Veneman, J.F., Ekkelenkamp, R., Kruidhof, R., van der Helm, F.C.T., van der Kooij, H.: A series elastic- and bowden-cable-based actuation system for use as a torque actuator in exoskeleton-type robots. International Journal of Robotics Research 25(3), 261-281 (2006)

17. Veneman, J.F., Kruidhof, R., Hekman, E.E.G., Ekkelenkamp, R., Van Asseldonk, E.H.F., van der Kooij, H.: Design and evaluation of the LOPES exoskeleton robot for interactive gait rehabilitation. IEEE Transactions on Neural Systems and Rehabilitation Engineering 15(3), 379-386 (2007)

18. van Asseldonk, E.H.F., Veneman, J.F., Ekkelenkamp, R., Buurke, J.H., van der Helm, F.C.T., van der Kooij, $\mathrm{H}$.: The effects on kinematics and muscle activity of walking in a robotic gait trainer during zero-force control. IEEE Transactions on Neural Systems and Rehabilitation Engineering 16(4), 360-370 (2008). doi:10.1109/TNSRE.2008.925074

19. Aurich-Schuler, T., Grob, F., van Hedel, H.A., Labruyère, R.: Can lokomat therapy with children and adolescents be improved? an adaptive clinical pilot trial comparing guidance force, path control, and FreeD. Journal of neuroengineering and rehabilitation 14(1), 76 (2017)

20. Aurich-Schuler, T., Gut, A., Labruyère, R.: The FreeD module for the lokomat facilitates a physiological movement pattern in healthy people-a proof of concept study. Journal of neuroengineering and rehabilitation 16(1), 26 (2019)

21. Jung, C.-Y., Choi, J., Park, S., Lee, J.M., Kim, C., Kim, S.-J.: Design and control of an exoskeleton system for gait rehabilitation capable of natural pelvic movement. In: Proceedings of the IEEE/RSJ International Conference on Intelligent Systems and Robots, Chicago, II., USA, pp. 2095-2100 (2014)

22. Son, C., Moon, H., Kim, D., Chun, M.H., Kim, S., Choi, J.: Effect of pelvic movement on healthy subjects during gait training using a gait rehabilitation system. In: Annual International Conference of the IEEE Engineering in Medicine and Biology Society (EMBC), Honolulu, HI., USA, pp. 2475-2478 (2018)

23. Jin, X., Cai, Y., Prado, A., Agrawal, S.K.: Effects of exoskeleton weight and inertia on human walking. 
Proceedings of IEEE International Conference on Robotics and Automation, 1772-1777 (2017). doi:10.1109/ICRA.2017.7989210

24. Rathore, A., Wilcox, M., Ramirez, D.Z.M., Loureiro, R., Carlson, T.: Quantifying the human-robot interaction forces between a lower limb exoskeleton and healthy users. In: Engineering in Medicine and Biology Society (EMBC), 2016 IEEE 38th Annual International Conference of The, pp. 586-589 (2016). IEEE

25. Jezernik, S., Colombo, G., Morari, M.: Automatic gait-pattern adaptation algorithm for rehabilitation with a 4-DOF robotic orthosis. IEEE Transactions on Robotics and Automation 20(3), 574-582 (2004)

26. Hidler, J.M., Wall, A.E.: Alterations in muscle activation patterns during robotic-assisted walking. Clinical Biomechanics 20(2), 184-193 (2005)

27. van Kammen, K., Boonstra, A.M., van der Woude, L.H.V., Visscher, C., Reinders-Messelink, H.A., den Otter, R.: Lokomat guided gait in hemiparetic stroke patients: the effects of training parameters on muscle activity and temporal symmetry. Disability and Rehabilitation 42(21), 2977-2985 (2019) doi:10.1080/09638288.2019.1579259

28. Kim, J.-H., Chung, Y., Kim, Y., Hwang, S.: Functional electrical stimulation applied to gluteus medius and tibialis anterior corresponding gait cycle for stroke. Gait \& posture 36(1), 65-67 (2012)

29. Kirker, S.G.B., Simpson, D.S., Jenner, J.R., Wing, A.M.: Stepping before standing: hip muscle function in stepping and standing balance after stroke. Journal of Neurology, Neurosurgery \& Psychiatry 68(4), 458-464 (2000)

30. Pandy, M.G., Andriacchi, T.P.: Muscle and joint function in human locomotion. Annual review of biomedical engineering 12, 401-433 (2010)

31. Lotze, M., Braun, C., Birbaumer, N., Anders, S., Cohen, L.G.: Motor learning elicited by voluntary drive. Brain 126(4), 866-872 (2003)

32. Veneman, J.F., Menger, J., van Asseldonk, E.H.F., van der Helm, F.C.T., van der Kooij, H.: Fixating the pelvis in the horizontal plane affects gait characteristics. Gait \& posture 28, 157-163 (2008) 


\section{Figures}

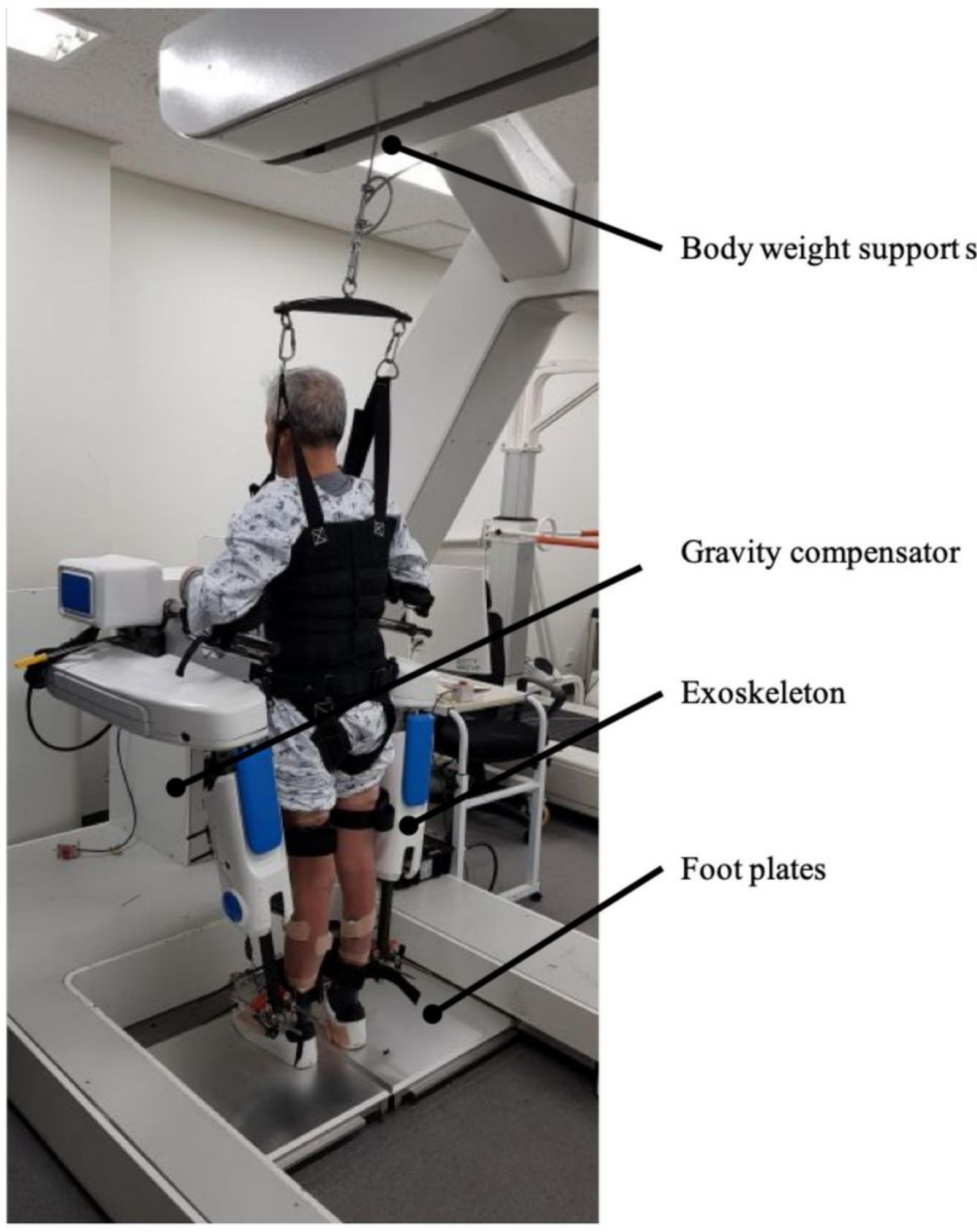

Figure 1

Subject in the Healbot T during training 

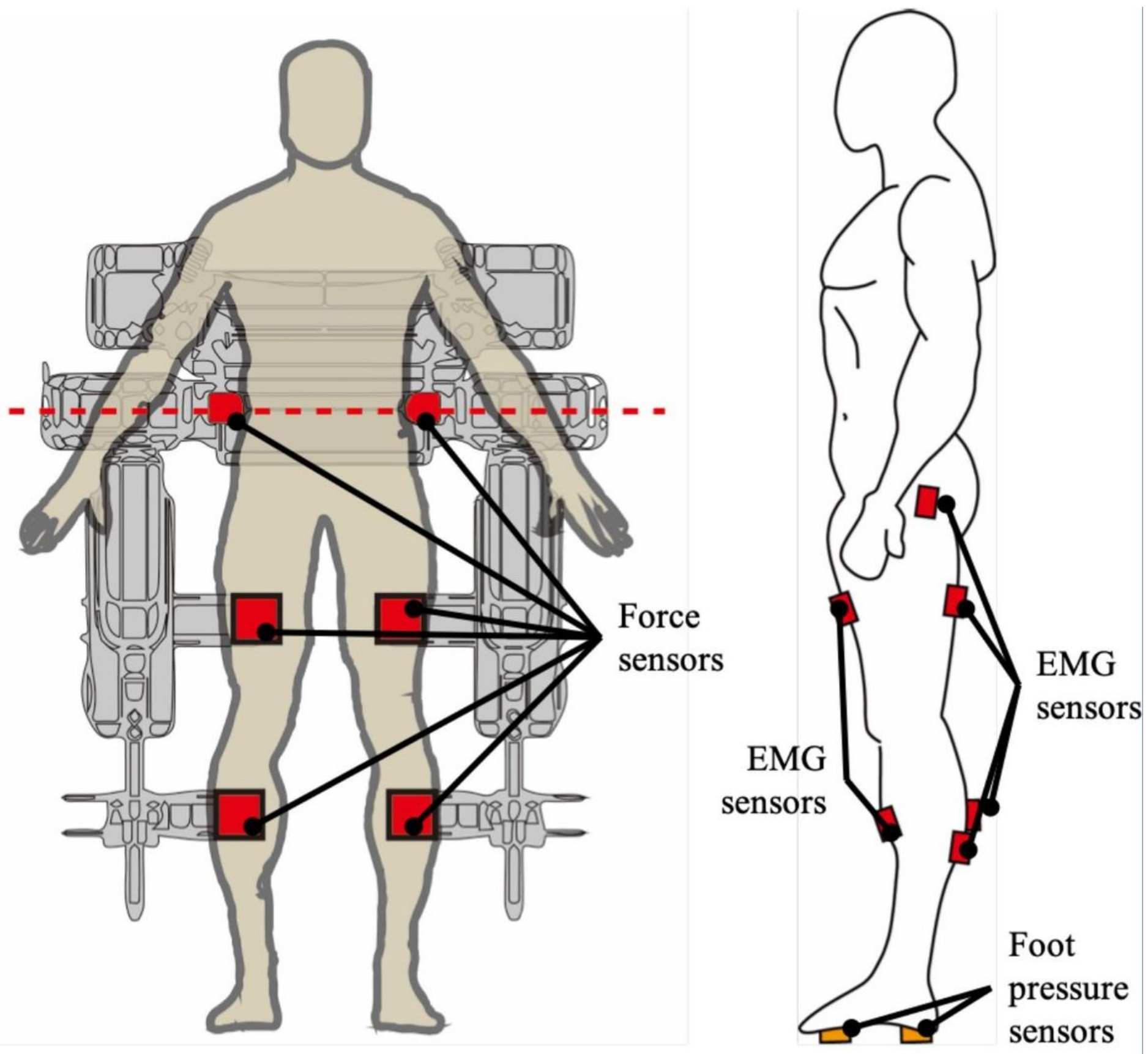

Figure 2

Locations of sensors. 6 force sensors are at the pelvis, thigh, and calf of each side. 12 EMG sensors are at the gluteus medius, rectus femoris, biceps femoris, tibialis anterior, gastrocnemius medial, gastrocnemius lateral. 2 pressure sensors are beneath each foot. 


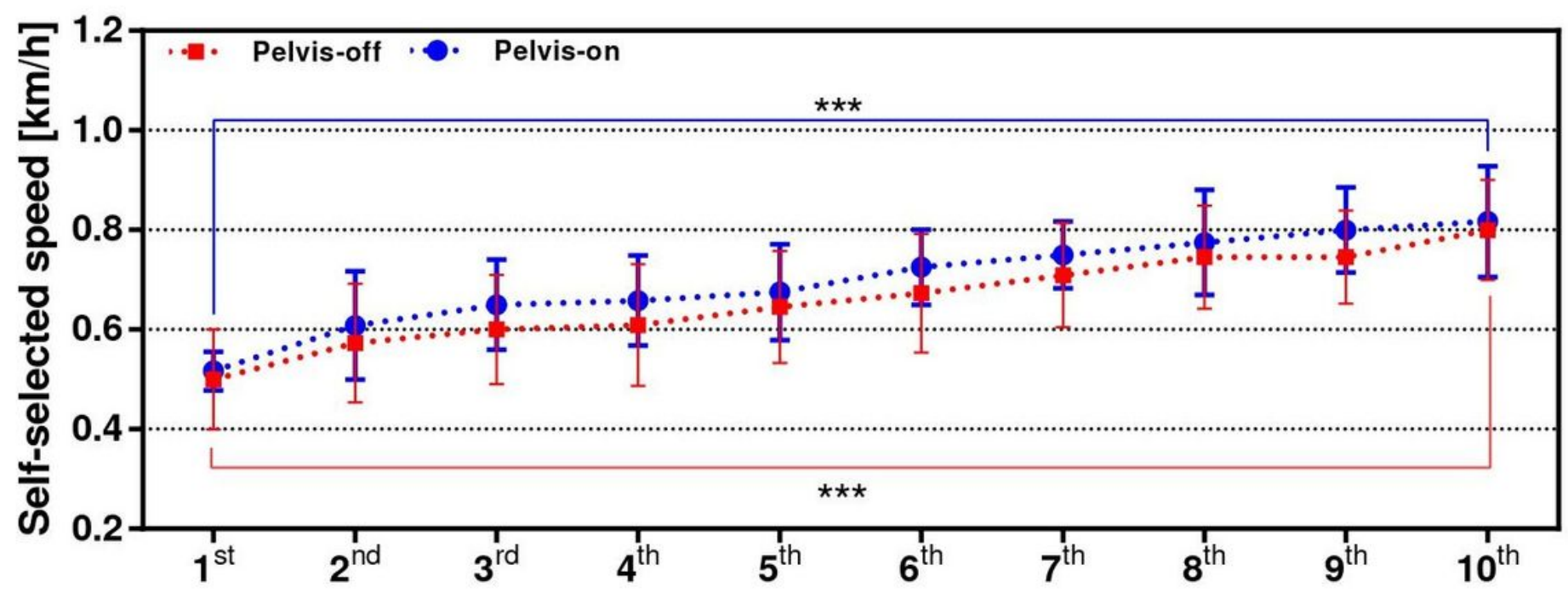

Figure 3

The self-selected speed of both groups. The red and blue lines represent the averaged self-selected speeds of the pelvis-off group and the pelvis-on group, respectively. 


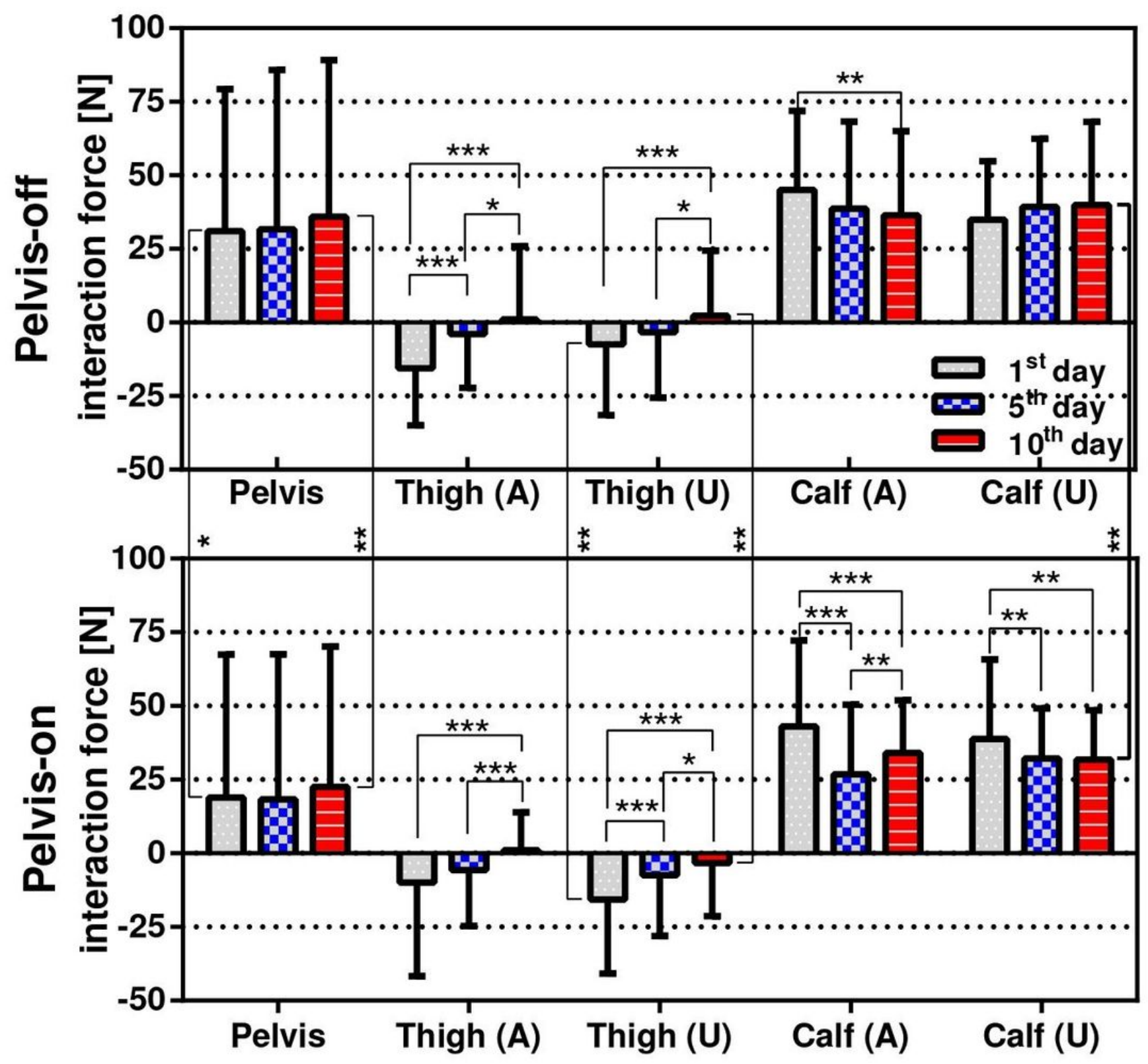

Figure 4

Measured interaction forces. The bar graphs represent mean of interaction force in both groups. The error bars represent standard deviation of interaction forces in both groups. 

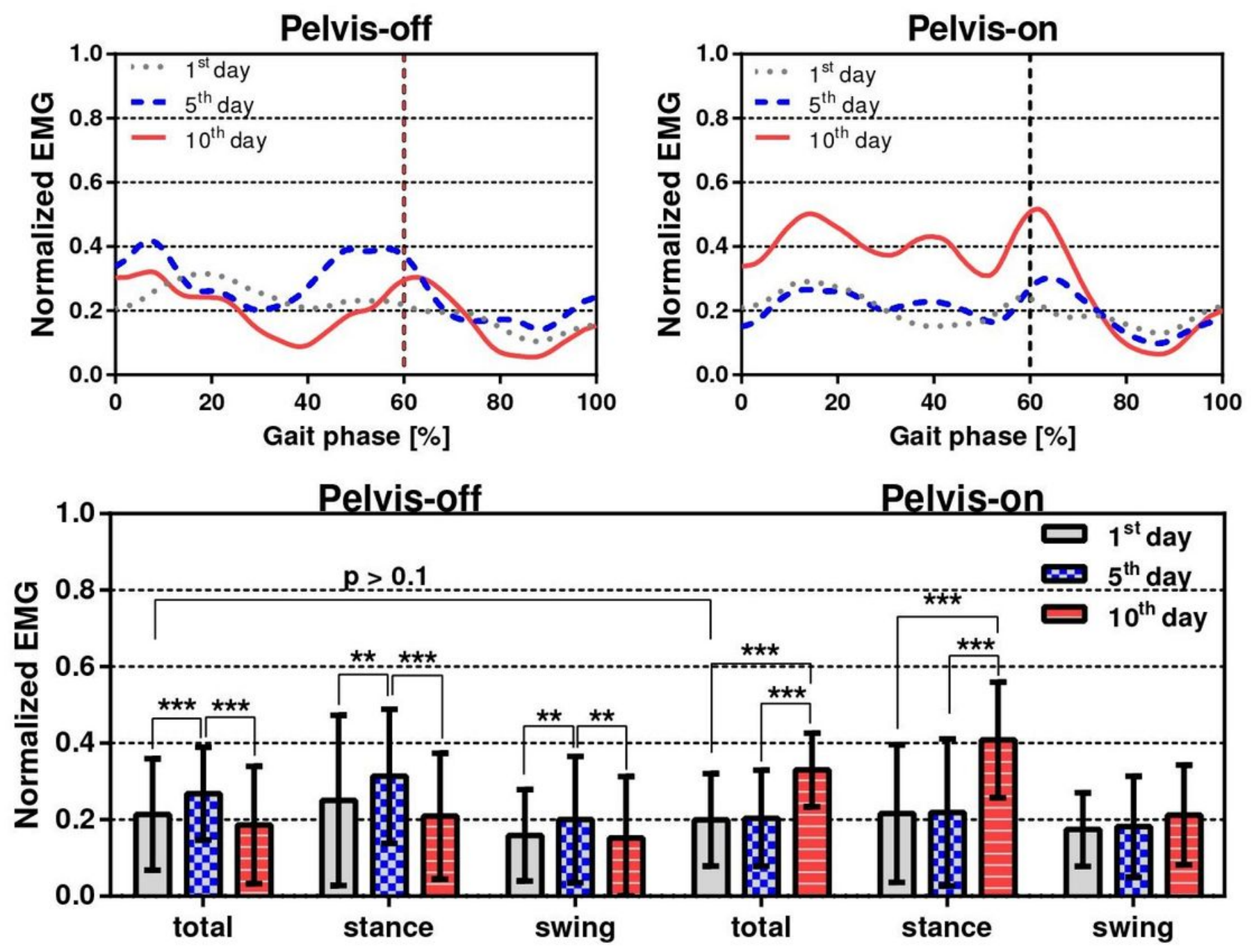

Figure 5

Normalized EMG records of Gmed on the affected side of both groups are at the top. Vertical dashed lines indicate the toe-off. The EMG records during different gait phases are at the bottom. The error bars represent standard deviation of EMG signal 

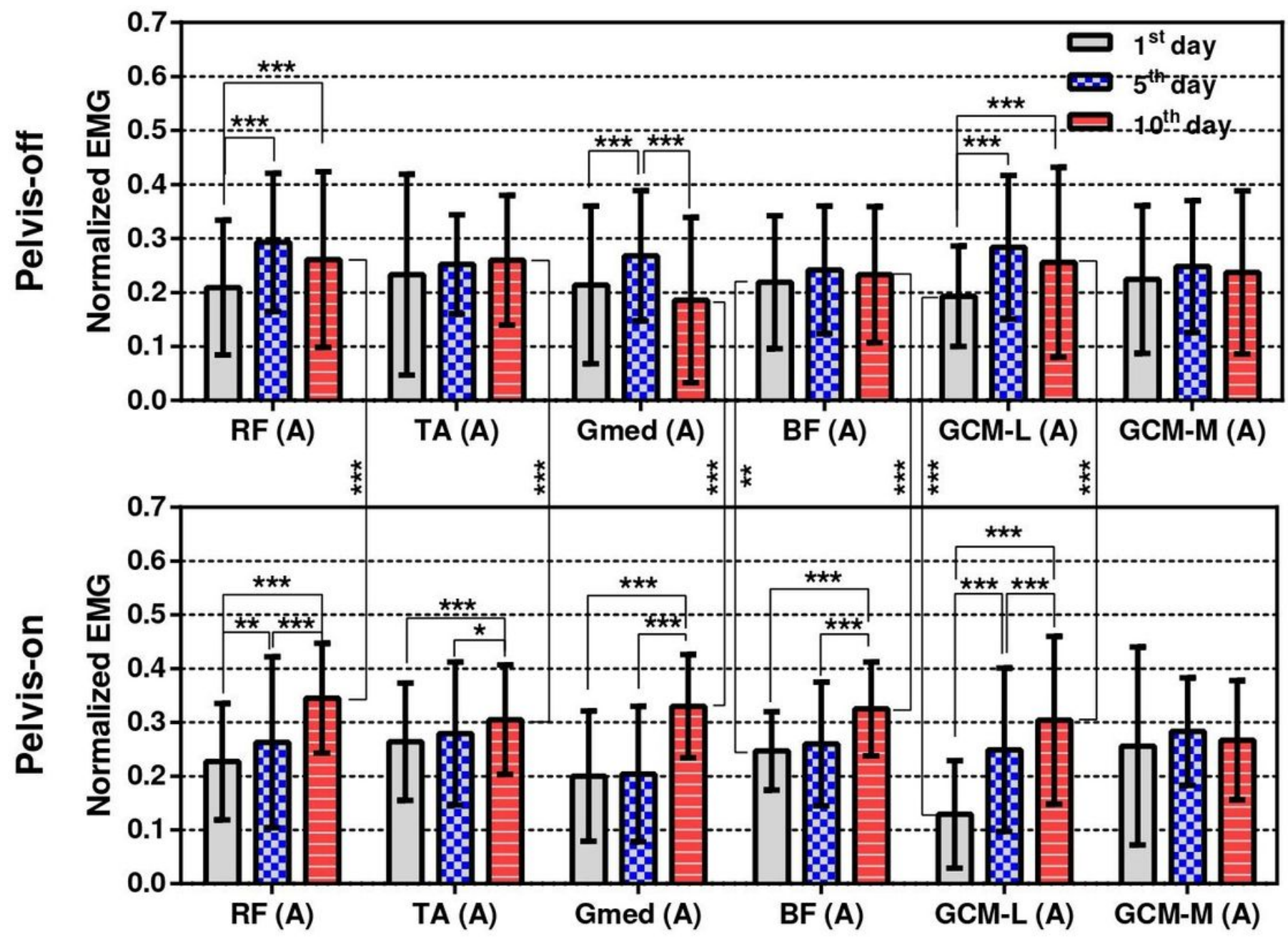

Figure 6

Averaged EMG signals of the 6 muscles on the affected sides of both groups. Top is the EMG signals of the pelvis-off group and bottom is the EMG signal of the pelvis-on group. 

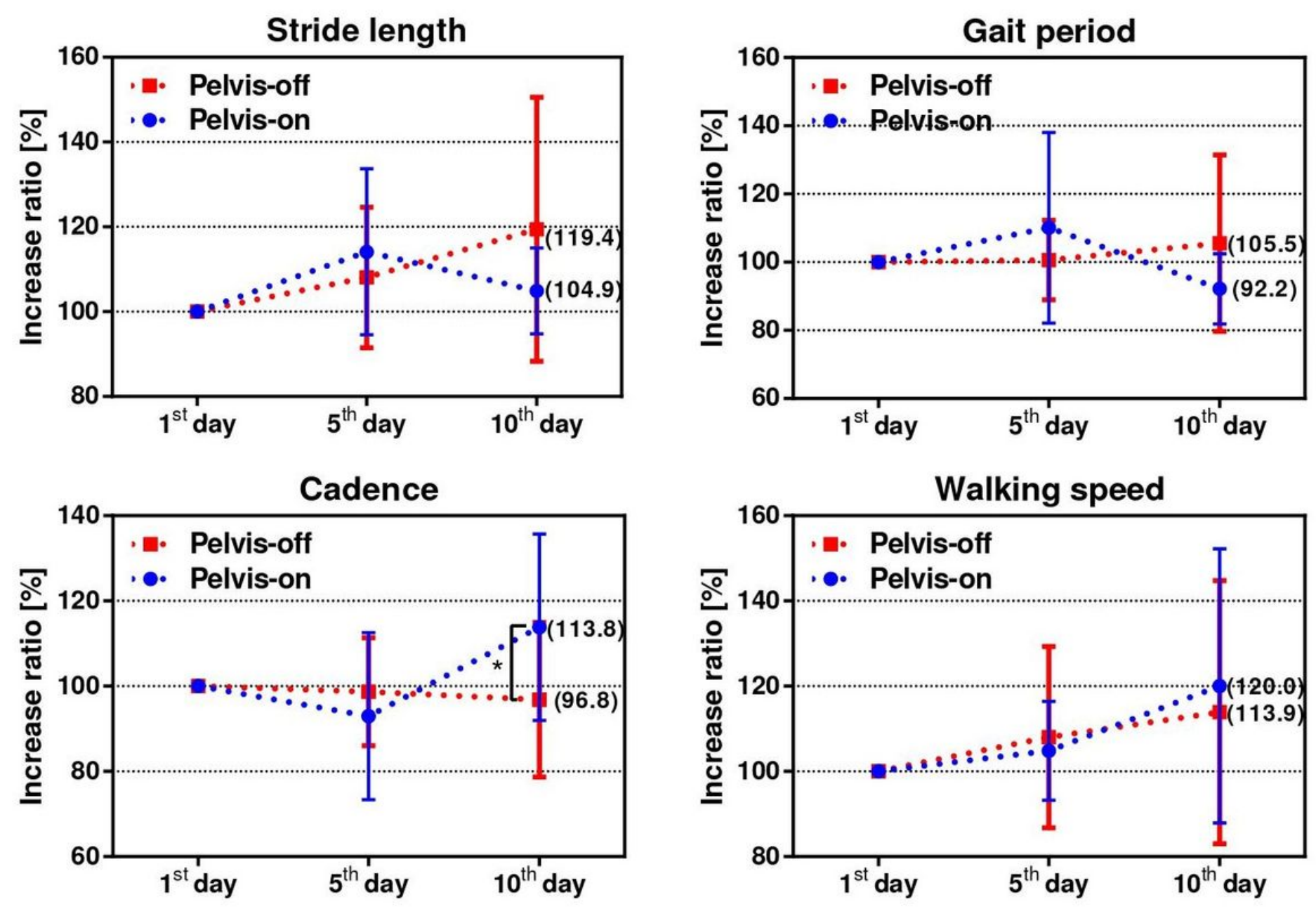

Figure 7

Measured gait parameters. Red and blue lines represent the pelvis-off group and the pelvis-on group. Error bars showed standard deviation. 


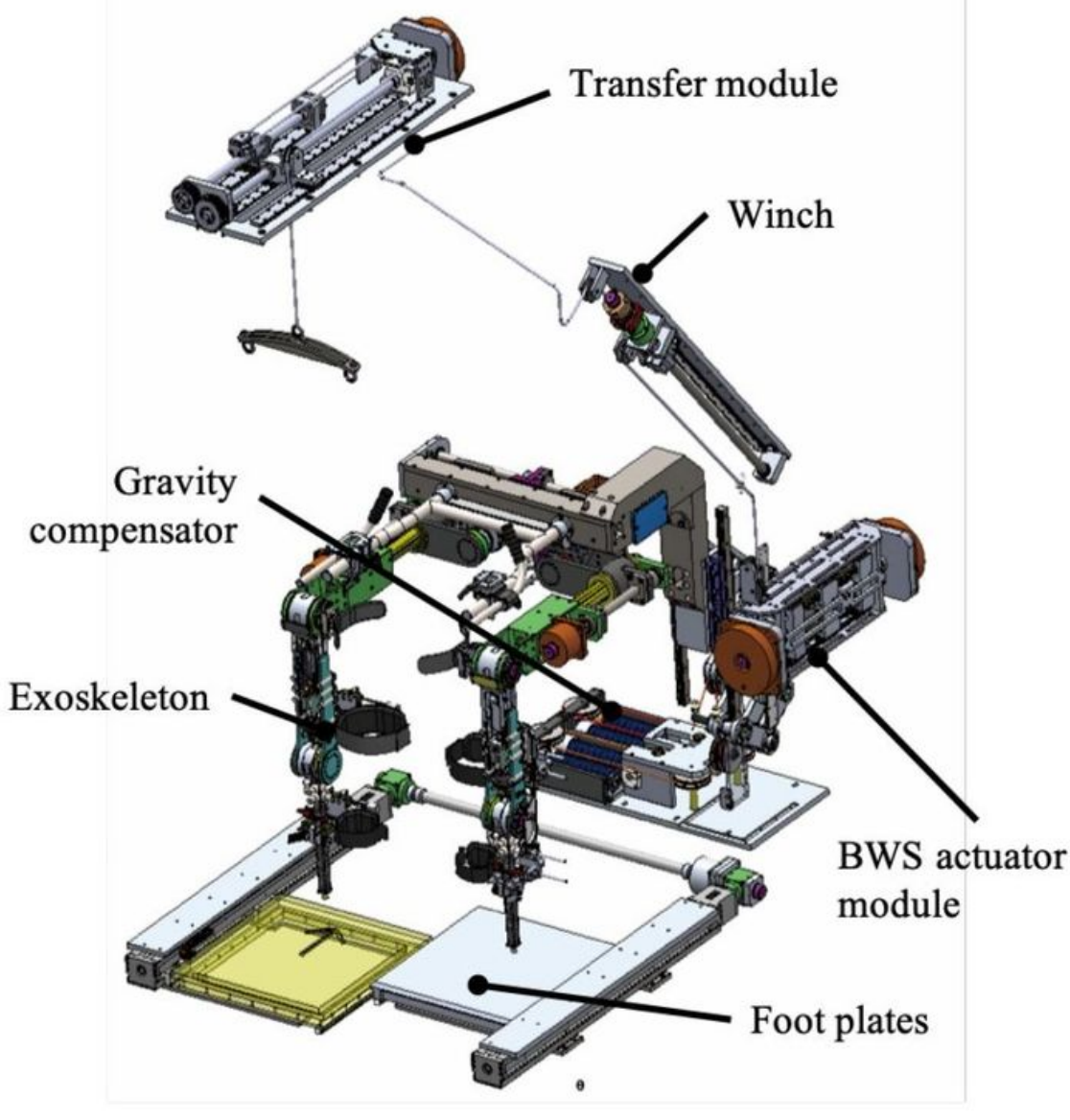

(a)

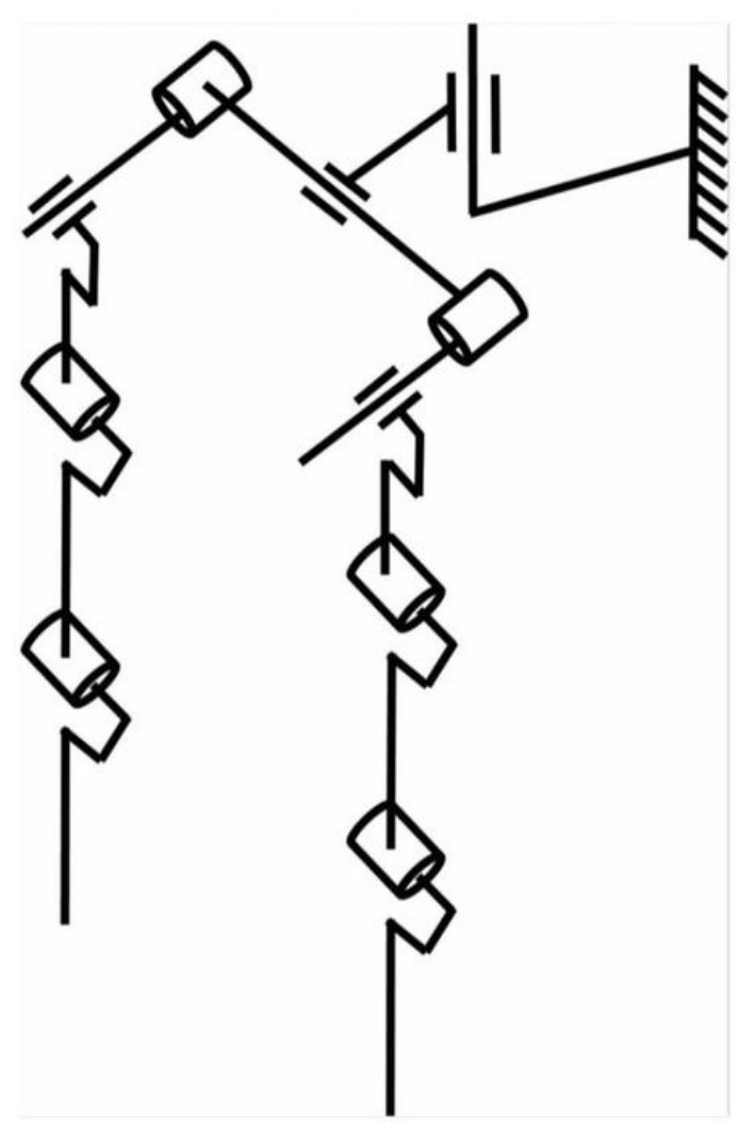

(b)

\section{Figure 8}

(a) Designed Healbot T. Healbot T consists of a lower limb exoskeleton robot with 9 active DoF and 1 passive DoF, a BWS system, a gravity compensator, and foot plates. (b) Schematics of Healbot T, which has 9 active joints and 1 passive joint for vertical displacement

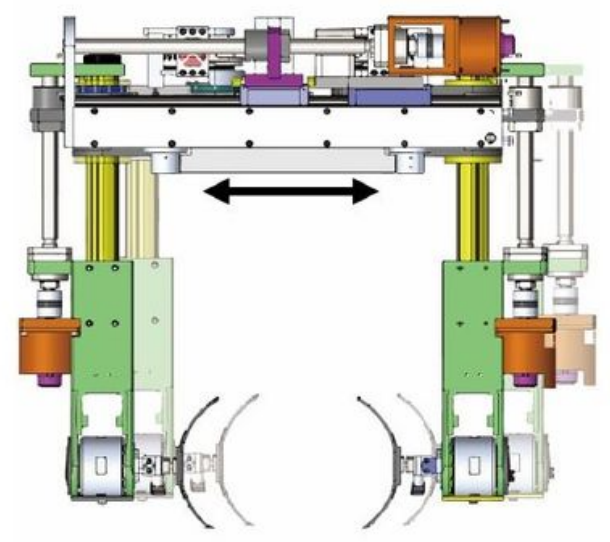

(a)

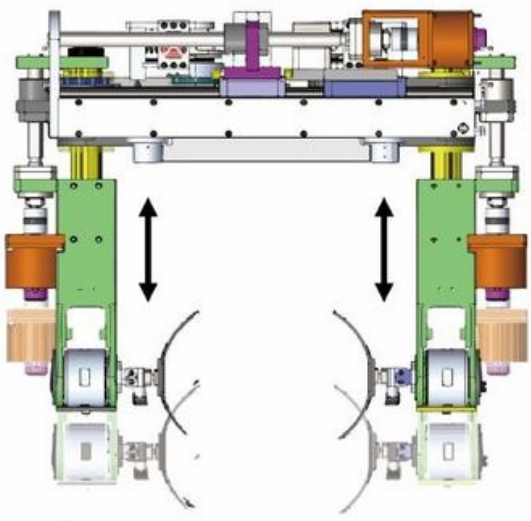

(b)

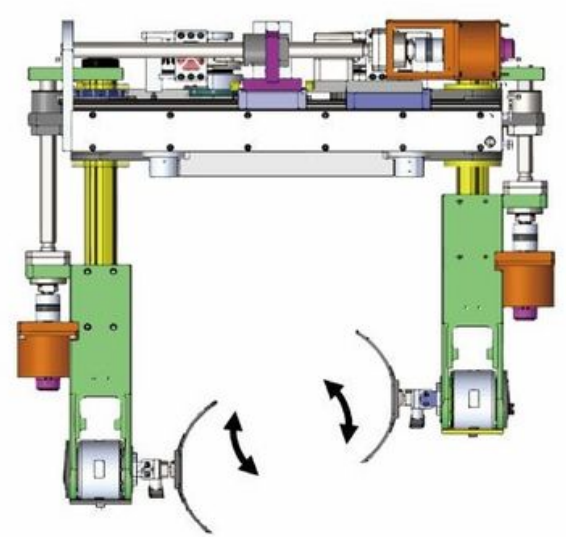

(c) 


\section{Figure 9}

Pelvic movement generated with three prismatic joints (a) Lateral movement (b) Forward movement (c) Rotational movement 